Fumi Murata-Mori, Naomi Hayashida, Takao Ando, Toshiyuki Ikeoka, Mio Nakazato, Harutaka Sekita, Norio Abiru, Hironori Yamasaki, Takahiro Maeda, Atsushi Kawakami and Noboru Takamura*

\title{
Association of the GCKR rs780094 polymorphism with metabolic traits including carotid intima-media thickness in Japanese community-dwelling men, but not in women
}

\begin{abstract}
Background: The glucokinase regulator gene (GCKR) rs780094 has been shown to be strongly associated with some metabolic traits and atherosclerotic parameters, while the association between GCKR rs780094 and carotid intima-media thickness (CIMT) has not been fully investigated in the general population. The associations between the GCKR rs780094 genotype and metabolic traits including CIMT were examined in a Japanese community-dwelling population.

Methods: A total of 2491 Japanese adults (907 men and 1584 women) who participated in a medical screening program for the general population from 29 to 94 years of age during 2008 to 2010 were enrolled. GCKR rs780094 was genotyped by the TaqMan polymerase chain reaction method, and associations with metabolic markers including CIMT were evaluated.

Results: GCKR rs780094 AA genotype was significantly associated with higher TG ( $\mathrm{p}<0.001 \mathrm{vs.} \mathrm{GG),} \mathrm{lower} \mathrm{HDL-C}$ ( $p=0.021$ vs. GG), and lower $\mathrm{HbA}_{1 \mathrm{c}}$ ( $\mathrm{p}=0.023$ vs. GG). The AA genotype showed significantly thinner CIMT ( $p=0.001$ vs. GX). These associations were seen only in men.

Conclusions: GCKR rs780094 was associated with TG, HDL-C, and $\mathrm{HbA}_{1 \mathrm{c}}$ levels, as well as with CIMT in Japanese community-dwelling men, but not women.
\end{abstract}

Keywords: carotid intima-media thickness; glucokinase regulator protein gene; sex difference; triglyceride; type 2 diabetes mellitus.

\footnotetext{
${ }^{*}$ Corresponding author: Noboru Takamura, MD, PhD, Professor and Chairman, Department of Global Health, Medicine and Welfare, Nagasaki University Graduate School of Biomedical Sciences, 1-12-4 Sakamoto, Nagasaki 852-8523, Japan, Phone: +8195 8197170, Fax: +81958197172, E-mail: takamura@nagasaki-u.ac.jp
}

Fumi Murata-Mori, Naomi Hayashida and Toshiyuki Ikeoka: Department of Global Health, Medicine and Welfare, Nagasaki University Graduate School of Biomedical Sciences, Nagasaki, Japan Takao Ando, Norio Abiru and Atsushi Kawakami: Department of Endocrinology and Metabolism, Unit of Translational Medicine, Nagasaki University Graduate School of Biomedical Sciences, Nagasaki, Japan Mio Nakazato, Harutaka Sekita and Takahiro Maeda: Department of Island and Community Medicine, Nagasaki University Graduate School of Biomedical Sciences, Nagasaki, Japan Hironori Yamasaki: Center for Health and Community Medicine, Nagasaki University, Nagasaki, Japan

\section{Introduction}

Glucokinase regulator protein (GKRP) is known to bind glucokinase (GCK) and suppress GCK's enzyme activity when fasting [1-3]. GCK plays a major role in blood glucose homeostasis not only by enhancing insulin secretion from the pancreatic $\beta$ cells, but also by regulating glycolysis and glycogen synthesis in the liver $[4,5]$. It has been shown that the GKRP-GCK complex in the nucleus dissociates postprandially, and then this dissociation allows GCK to translocate from the nucleus to the cytoplasm, where GCK becomes an active form [2, 3, 5, 6]. Therefore, GKRP plays a major role in regulating glucose homeostasis as a GCK modulator.

GKRP is encoded by glucokinase regulator protein gene $(G C K R)$. It has been recently shown that novel single nucleotide polymorphisms (SNPs) of rs780094 in the GCKR are candidate susceptibility variants in association with type 2 diabetes mellitus (T2DM) [7]. This SNP, rs780094, is located at intron 16 and has been shown to have strong linkage disequilibrium $\left(\mathrm{r}^{2}=0.932\right)$ with another SNP, rs1260326 (a non-synonymous SNP, 
Pro446Leu). In several genome-wide association studies, the derived "A" allele of rs780094 was found to be associated with several metabolic phenotypes in various ethnic groups. These phenotypes included higher triglycerides, reduced risk of T2DM, lower fasting glucose, lower fasting insulin, lower HOMA-IR, lower HOMA- $\beta$, higher 2-h glucose on the oral glucose tolerance test, higher C-reactive protein, and increased risk of non-alcoholic fatty liver disease [4, 8-15].

Despite the association of GCKR rs780094 with several metabolic traits, the contribution of the SNP to the development of atherosclerosis has been studied only in subjects with T2DM and metabolic syndrome [16]. Whether GCKR rs780094 contributes to the development of atherosclerosis in the general population remains unknown. Since CIMT is a surrogate marker of subclinical atherosclerosis [17] and a predictor of stroke and cardiovascular disease [17-19], the associations of GCKR rs780094 with metabolic traits and carotid intima-media thickness (CIMT) were evaluated in a Japanese community-dwelling population.

\section{Materials and methods}

\section{Study population}

Prior to this study, ethical approval was obtained from the Special Committee of Nagasaki University (project registration number 0501120073). This study was conducted during a medical screening program for the general population residing in Goto City, Nagasaki Prefecture, Japan. All data were collected by the staff of Nagasaki University, in cooperation with the staff of Goto City. After obtaining informed consent, 2581 Japanese adults ranging in age from 29 to 94 years who participated in the medical screening program performed by Goto City during the period from 2008 to 2010 were enrolled. Fourteen participants with marked hypertriglyceridemia ( $\geq 4.0 \mathrm{~g} / \mathrm{L}$ ) were excluded from the study, since low-density lipoprotein-cholesterol (LDL-C) could not be calculated using the Friedewald equation [20]. Seventy-six participants were excluded due to incomplete data. Finally, 2491 participants (907 men, 1584 women) were included in the analysis. The study participants were mainly middle-aged to elderly, because employees, which include relatively young generation, underwent other medical checkups at their workplaces in Japan. Overall, 93\% of the participants were more than 50 years old, and $76 \%$ of the participants were more than 60 years old.

Using software for sample size calculation (CaTS ${ }^{\circledR}$, http:// www.sph.umich.edu/csg/abecasis/CaTS/index.html), it was confirmed that the sample size (both men and women) was sufficient to identify the association between GCKR rs780094 polymorphism and metabolic traits including CIMT in the study population. The clinical characteristics of the study participants are summarized in Table 1.

\section{Data collection and laboratory measurements}

The height and weight of each participant were measured, and the body mass index (BMI, $\mathrm{kg} / \mathrm{m}^{2}$ ) was calculated as an index of obesity. Waist circumference was measured horizontally at the umbilicus with a tape measure after normal expiration. Systolic blood pressure (SBP) and diastolic blood pressure (DBP) were recorded at rest.

Blood samples were collected from each participant after an overnight fast. Serum and plasma obtained were separated and stored at $-20^{\circ} \mathrm{C}$ and $-80^{\circ} \mathrm{C}$, respectively. Serum concentrations of total cholesterol (TC), triglycerides (TG), and high-density lipoprotein-cholesterol (HDL-C) were measured using standard laboratory procedures. LDL-C was calculated using the Friedewald equation. In addition, serum creatinine (Cre), uric acid (UA), and glycated hemoglobin $\left(\mathrm{HbA}_{1 \mathrm{c}}\right)$ were measured using standard laboratory procedures. The conversion equation from $\mathrm{HbA}_{1 \mathrm{c}}$ (Japan Diabetes Society) to $\mathrm{HbA}_{1 \mathrm{c}}$ (National Glycohemoglobin Standardization Program) units officially certified by the Japan Diabetes Society was used [21]. Estimated glomerular filtration rate (eGFR) was calculated by serum Cre, sex, and age using the formula: $194 \times \mathrm{Cre}^{-1.094} \times \mathrm{Age}^{-0.287}$ for men and then multiplied by 0.739 for women.

Three physicians (N.H., M.N., and N.T.) measured CIMT by ultrasonography of the right and left carotid arteries using a LOGIC Book $\mathrm{XP}$ with a 10-MHz linear array transducer (GE Medical Systems, Milwaukee, WI, USA). Intra-observer variation of CIMT (N.T., $\mathrm{n}=32$ ) was $0.91(\mathrm{p}<0.01)$, and inter-observer variation (N.T. vs. M.N., $\mathrm{n}=41)$ was $0.78(\mathrm{p}<0.01)$. The far wall of the carotid artery was displayed on a longitudinal two-dimensional ultrasonographic image as two bright white lines separated by a hypoechoic space. The distance from the leading edge of the first (lumen-intima interface) to the leading edge of the second (media-adventitia interface) bright line was defined as the CIMT. Images were analyzed using Intima Scope ${ }^{\odot}$ software (MEDIA CROSS, Tokyo, Japan). The average of the right and left CIMTs was calculated and used in the analysis.

\section{Genotyping}

Genomic DNA was automatically extracted from blood cells separated from plasma using a MagExtractor MFX ${ }^{\circledR}$ (TOYOBO, Osaka, Japan). For the determination of GCKR rs780094 genotypes, the TaqMan polymerase chain reaction method (Applied Biosystems Japan, Tokyo, Japan) was used. In this study, two probes were prepared: the "G" allele-specific probe, 5' - VIC - GTT TTT TAG ACC ATG ACT GAC ACA TGT TTG CTG ATC AAT ACA TTT GTT GAG-Tamra - 3 ' and the "A" allele-specific probe, 5' - FAM - GTT TTT TAG ACC ATG ACT GAC ACA TAT TTG CTG ATC AAT ACA TTT GTT GAG -Tamra - $3^{\prime}$. The primer design for polymerase chain reaction of the flanking region of GCKR rs780094 was as follows: forward, 5' - GCT TCT TGA AAG GGC AGA GA - 3'; reverse, 5' - GGA TCA CCT GAG GTC AGG AG - 3'. The polymerase chain reaction was carried out with a thermal cycler (BioRad Laboratories, Berkeley, CA, USA) according to the following conditions: initial denaturation at $95^{\circ} \mathrm{C}$ for $10 \mathrm{~min}$, followed by 35 cycles at $95^{\circ} \mathrm{C}$ for $30 \mathrm{~s}$ for denaturation, $55^{\circ} \mathrm{C}$ for $1 \mathrm{~min}$ for annealing, and $72^{\circ} \mathrm{C}$ for $30 \mathrm{~s}$ for primer extension. Final extension was performed at $72^{\circ} \mathrm{C}$ for $10 \mathrm{~min}$. The fluorescence level of polymerase chain reaction products was measured with an ABI PRISM 7900 Sequence Detector 
Table 1 Characteristics of study participants.

\begin{tabular}{|c|c|c|c|}
\hline & Men $(n=907)$ & Women $(n=1584)$ & Total $(n=2491)$ \\
\hline Age, years & $67.3 \pm 10.6$ & $66.4 \pm 10.6$ & $66.7 \pm 10.6$ \\
\hline Height, cm & $162.9 \pm 6.6$ & $150.7 \pm 6.3$ & $155.1 \pm 8.7$ \\
\hline Weight, kg & $62.5 \pm 9.6$ & $52.0 \pm 8.5$ & $55.8 \pm 10.2$ \\
\hline Waist circumference, $\mathrm{cm}$ & $84.6 \pm 8.6$ & $82.6 \pm 10.1$ & $83.3 \pm 9.7$ \\
\hline $\mathrm{BMI}, \mathrm{kg} / \mathrm{m}^{2}$ & $23.5 \pm 3.1$ & $22.9 \pm 3.4$ & $23.1 \pm 3.3$ \\
\hline SBP, mm Hg & $144.0 \pm 20.4$ & $141.7 \pm 22.2$ & $142.5 \pm 21.6$ \\
\hline DBP, $\mathrm{mm} \mathrm{Hg}$ & $84.3 \pm 11.0$ & $82.0 \pm 11.0$ & $82.9 \pm 11.0$ \\
\hline Cre, mg/L & 8.6. \pm 2.0 & $6.4 \pm 1.4$ & $7.2 \pm 2.0$ \\
\hline $\mathrm{eGFR}, \mathrm{mL} / \mathrm{min} / 1.73 \mathrm{~m}^{2}$ & $72.6 \pm 16.3$ & $73.6 \pm 15.1$ & $73.2 \pm 15.6$ \\
\hline $\mathrm{UA}, \mathrm{g} / \mathrm{L}$ & $0.06 \pm 0.01$ & $0.05 \pm 0.01$ & $0.05 \pm 0.01$ \\
\hline $\mathrm{TC}, \mathrm{g} / \mathrm{L}$ & $1.91 \pm 0.33$ & $2.07 \pm 0.34$ & $2.01 \pm 0.34$ \\
\hline $\mathrm{TG}, \mathrm{g} / \mathrm{L}$ & $0.85(0.61-1.26)$ & $0.86(0.64-1.19)$ & $0.86(0.63-1.21)$ \\
\hline HDL-C, g/L & $0.56 \pm 0.14$ & $0.62 \pm 0.15$ & $0.60 \pm 0.15$ \\
\hline LDL-C, g/L & $1.15 \pm 0.29$ & $1.26 \pm 0.30$ & $1.22 \pm 0.30$ \\
\hline LDL-C/HDL-C ratio & $2.2 \pm 0.8$ & $2.2 \pm 0.8$ & $2.2 \pm 0.8$ \\
\hline Non-HDL-C, g/L & $1.35 \pm 0.33$ & $1.45 \pm 0.34$ & $1.42 \pm 0.34$ \\
\hline Glu, g/L & $1.02 \pm 0.27$ & $0.97 \pm 0.19$ & $0.99 \pm 0.22$ \\
\hline $\mathrm{HbA}_{1 \mathrm{c}}, \%$ & $5.69 \pm 0.68$ & $5.67 \pm 0.50$ & $5.68 \pm 0.57$ \\
\hline Max IMT, mm & $0.97 \pm 0.21$ & $0.91 \pm 0.19$ & $0.93 \pm 0.20$ \\
\hline Mean IMT, mm & $0.75 \pm 0.14$ & $0.71 \pm 0.13$ & $0.72 \pm 0.14$ \\
\hline Current smoker, n (\%) & $189(20.8)$ & $38(2.4)$ & $227(9.1)$ \\
\hline Medication for HT, n (\%) & $310(34.2)$ & $539(34.0)$ & $849(34.1)$ \\
\hline Medication for DM, n (\%) & $74(8.2)$ & $63(4.0)$ & $137(5.5)$ \\
\hline Medication for DL, n (\%) & $73(8.0)$ & $230(14.5)$ & $303(12.2)$ \\
\hline IHD, n (\%) & $51(5.6)$ & $36(4.0)$ & $114(4.6)$ \\
\hline CVD, n (\%) & $31(3.4)$ & $34(2.1)$ & $65(2.6)$ \\
\hline
\end{tabular}

Values are means \pm SD, medians (25th-75th), or the percentage of the participants with the particular habit or clinical disorders. BMI, body mass index; CIMT, carotid intima-media thickness; CRE, creatinine; CVD, cerebrovascular disease; DL, dyslipidemia; DM, diabetes mellitus; $\mathrm{HbA}_{1 \mathrm{c}}$, hemoglobin $\mathrm{A}_{1 \mathrm{c}}$; HDL-C, high-density lipoprotein cholesterol; HT, hypertension; IHD, ischemic heart disease; LDL-C, low-density lipoprotein cholesterol; MBP, mean blood pressure; TC, total cholesterol; TG, triglyceride; UA, uric acid.

(Applied Biosystems Japan) to identify three GCKR rs780094 genotypes (GG, GA, and AA).

\section{Statistical analysis}

The results are expressed as means \pm standard deviation or medians (25th-75th quartile). Differences of laboratory values between men and women were evaluated using the t-test and the Mann-Whitney U-test. Because TG was distributed in a skewed manner, logarithmic transformation was performed for the analysis. Differences in smoking status (current smoker vs. non-smoker including past smoker), the rate of current treatment for hypertension (HT), diabetes mellitus (DM), and dyslipidemia (DL), and the rate of past history of ischemic heart disease (IHD) and cerebrovascular disease (CVD) were evaluated using the $\chi^{2}$-test. The differences in each value among the GCKR rs780094 genotypes were evaluated using analysis of variance. Furthermore, the effects of GCKR rs780094 on CIMT were evaluated by analysis of covariance after adjustment for age and other confounding factors such as BMI, SBP, $\log \mathrm{TG}, \mathrm{HbA}_{1 \mathrm{c}}$, smoking status, and history of current medications for HT, DL, and DM. Probability values below 0.05 were considered significant. All data were statistically analyzed using SPSS ver 18.0 software (SPSS Japan, Tokyo, Japan).

\section{Results}

The distribution of the GCKR rs780094 was consistent with Hardy-Weinberg equilibrium, and no significant differences were observed in the allele frequencies of AA and GX (GA and GG) between men and women (71.6\% vs. $28.3 \%$ in men and $74.1 \%$ vs. $25.9 \%$ in women, respectively).

The associations of genotype GCKR rs780094 with metabolic traits and CIMT were evaluated. All subjects have been divided between GX (GG and GA) and AA; it shows that the GX genotype was not significantly related to CIMT, and some parameters were significantly different between AA and other genotypes (Table 2). Since age and sex are classical risk factors and are important for development of metabolic traits and/or atherosclerosis, data were then analyzed by adjusting for age separately, by sex. The AA genotype showed significantly higher $\log \mathrm{TG}(\mathrm{p}<0.001$ vs. $\mathrm{GG}, \mathrm{p}=0.004$ vs. $\mathrm{GA})$, lower HDL-C ( $p=0.021$ vs. GG), and lower $\operatorname{HbA}_{1 c}(p=0.023$ vs. $\mathrm{GG}$ ) in men; these associations were, however, not seen 
Table 2 Clinical phenotypes according to GCKR genotypes adjusted for age.

\begin{tabular}{|c|c|c|c|}
\hline Phenotype & GG & GA & AA \\
\hline \multicolumn{4}{|l|}{ BMI } \\
\hline All & $23.1 \pm 0.1$ & $23.1 \pm 0.1$ & $23.2 \pm 0.1$ \\
\hline Men & $23.5 \pm 0.2$ & $23.4 \pm 0.2$ & $23.5 \pm 0.2$ \\
\hline Women & $22.8 \pm 0.2$ & $22.8 \pm 0.1$ & $23.0 \pm 0.2$ \\
\hline \multicolumn{4}{|l|}{ SBP } \\
\hline All & $142.6 \pm 0.8$ & $142.8 \pm 0.6$ & $141.8 \pm 0.8$ \\
\hline Men & $145.2 \pm 1.3$ & $144.1 \pm 1.0$ & $142.7 \pm 1.2$ \\
\hline Women & $141.1 \pm 1.1$ & $142.0 \pm 0.7$ & $141.4 \pm 1.0$ \\
\hline \multicolumn{4}{|l|}{ DBP } \\
\hline All & $82.8 \pm 0.5$ & $82.9 \pm 0.3$ & $82.9 \pm 0.4$ \\
\hline Men & $84.4 \pm 0.7$ & $84.7 \pm 0.5$ & $83.8 \pm 0.7$ \\
\hline Women & $82.0 \pm 0.6$ & $81.9 \pm 0.4$ & $82.4 \pm 0.5$ \\
\hline \multicolumn{4}{|l|}{$\log \mathrm{TG}$} \\
\hline All & $1.93 \pm 0.01$ & $1.94 \pm 0.01$ & $1.97 \pm 0.01^{\mathrm{b}, \mathrm{d}}$ \\
\hline Men & $1.92 \pm 0.01$ & $1.94 \pm 0.01$ & $1.99 \pm 0.01^{\mathrm{c}, \mathrm{d}}$ \\
\hline Women & $1.94 \pm 0.01$ & $1.94 \pm 0.01$ & $1.96 \pm 0.01$ \\
\hline \multicolumn{4}{|l|}{ HDL-C } \\
\hline All & $0.60 \pm 0.01$ & $0.59 \pm 0.00$ & $0.59 \pm 0.01$ \\
\hline Men & $0.57 \pm 0.01$ & $0.56 \pm 0.01$ & $0.54 \pm 0.01^{\mathrm{a}}$ \\
\hline Women & $0.62 \pm 0.01$ & $0.62 \pm 0.00$ & $0.62 \pm 0.01$ \\
\hline \multicolumn{4}{|l|}{ LDL-C } \\
\hline All & $1.23 \pm 0.01$ & $1.21 \pm 0.01$ & $1.22 \pm 0.01$ \\
\hline Men & $1.17 \pm 0.02$ & $1.14 \pm 0.01$ & $1.15 \pm 0.02$ \\
\hline Women & $1.26 \pm 0.02$ & $1.25 \pm 0.01$ & $1.26 \pm 0.02$ \\
\hline \multicolumn{4}{|l|}{ Glu } \\
\hline All & $1.00 \pm 0.01$ & $0.99 \pm 0.01$ & $0.98 \pm 0.01$ \\
\hline Men & $1.01 \pm 0.02$ & $1.04 \pm 0.01$ & $0.99 \pm 0.02$ \\
\hline Women & $1.00 \pm 0.01$ & $0.96 \pm 0.01^{\mathrm{a}}$ & $0.97 \pm 0.01^{\mathrm{a}}$ \\
\hline \multicolumn{4}{|l|}{$\mathrm{HbA}_{1 \mathrm{c}}$} \\
\hline All & $5.71 \pm 0.02$ & $5.68 \pm 0.02$ & $5.66 \pm 0.02$ \\
\hline Men & $5.76 \pm 0.04$ & $5.69 \pm 0.03$ & $5.62 \pm 0.04^{\mathrm{a}}$ \\
\hline Women & $5.67 \pm 0.03$ & $5.67 \pm 0.02$ & $5.68 \pm 0.03$ \\
\hline \multicolumn{4}{|l|}{ Max CIMT } \\
\hline All & $0.93 \pm 0.01$ & $0.93 \pm 0.01$ & $0.92 \pm 0.01$ \\
\hline Men & $0.96 \pm 0.01$ & $0.98 \pm 0.01$ & $0.94 \pm 0.01^{\mathrm{d}}$ \\
\hline Women & $0.92 \pm 0.01$ & $0.91 \pm 0.01$ & $0.91 \pm 0.01$ \\
\hline \multicolumn{4}{|l|}{ Mean CIMT } \\
\hline All & $0.72 \pm 0.01$ & $0.72 \pm 0.00$ & $0.72 \pm 0.01$ \\
\hline Men & $0.75 \pm 0.01$ & $0.76 \pm 0.01$ & $0.72 \pm 0.01^{\mathrm{d}}$ \\
\hline Women & $0.71 \pm 0.01$ & $0.70 \pm 0.00$ & $0.71 \pm 0.01$ \\
\hline
\end{tabular}

${ }^{a} p<0.05,{ }^{b} p<0.01,{ }^{c} p<0.001$ vs. GG, ${ }^{d} p<0.001$ vs. GA. Data are shown as means \pm SE and were evaluated using analysis of covariance (ANCOVA).

in women. In terms of atherosclerosis, the AA genotype was associated with thinner maximum CIMT ( $p=0.001$ vs. GA) and thinner mean CIMT ( $\mathrm{p}=0.001$ vs. GA) than the GX genotypes in men; these associations were, however, not seen in women, as for the metabolic traits (Table 2). Since these parameters, such as $\log \mathrm{TG}$, HDL-C, and $\mathrm{HbA}_{1 \mathrm{c}}$, might be confounding factors to CIMT, these parameters were adjusted to determine the contribution of GCKR rs780094 to CIMT. The associations of GCKR rs780094 with maximum and mean CIMTs remained significant even when adjusted for age and BMI (Table 3, model 1), when adjusted for age, BMI, and metabolic traits (Table 3, model 2), and when adjusted for age, BMI, metabolic traits, and smoking status (Table 3, model 3). Moreover, the AA genotype of GCKR rs780094 still remained associated with thinner maximum and mean CIMTs, even when adjusted for age, BMI, metabolic traits, smoking, and treatment with antihypertensive, lipid lowering, and hypoglycemic drugs (Table 3, model 4). However, these associations were seen only in men, not in women.

\section{Discussion}

In this study, the AA genotype of GCKR rs780094 was significantly associated with higher serum TG, lower serum HDL-C, lower plasma Glu, and lower $\mathrm{HbA}_{1 \mathrm{c}}$ than the GX genotype in men. Importantly, this is the first time that the AA genotype, unlike the GX genotype, was found to be independently associated with thinner maximum and mean CIMTs in men. In contrast, no association was found in women.

The finding of the association of the AA genotype with higher serum TG was not surprising. Sparsø et al. and Stančáková et al. recently reported that the same SNP was associated with increased TG levels [4] and increased VLDL particle concentrations [22]. The present observation of the association of the AA genotype with decreased plasma glucose and $\mathrm{HbA}_{1 \mathrm{c}}$ levels was consistent with the previous report by Spars $\varnothing$ et al. showing lower fasting plasma glucose in the AA genotype [4].

To the best of our knowledge, the association between the GCKR rs780094 and CIMT in the general population was found for the first time. CIMTs were significantly thinner in men with the AA genotype of GCKR rs780094 than in those with the GX genotype. In contrast, Mohás et al. showed that the AA genotype of GCKR rs780094 was associated with thicker CIMT in Hungarian patients with metabolic syndrome $(n=455)$, but not significantly associated in patients with T2DM ( $\mathrm{n}=321)$. Unfortunately, they did not evaluate CIMT in healthy controls $(n=172)$ [16]. The discrepancy between Mohás's study and the present study may be due to the different type of subjects (metabolic syndrome vs. general population, and different racial background) or the number of participants. Further studies are needed to establish the association between GCKR polymorphisms and CIMT. 
Table 3 Association of GCKR rs780094 genotypes with CIMT with adjustments for several metabolic traits.

\begin{tabular}{|c|c|c|c|c|c|c|}
\hline Phenotype & $\log$ TG & HDL-C, g/L & Glu, g/L & $\mathrm{HbA}_{1 \mathrm{c}}, \%$ & Max CIMT, mm & Mean CIMT, mm \\
\hline \multicolumn{7}{|l|}{ Model 1} \\
\hline \multicolumn{7}{|l|}{ All } \\
\hline $\mathrm{GX}$ & $1.94 \pm 0.01^{\mathrm{b}}$ & $0.60 \pm 0.00$ & $0.99 \pm 0.01$ & $5.69 \pm 0.01$ & $0.93 \pm 0.00$ & $0.72 \pm 0.00$ \\
\hline $\mathrm{AA}$ & $1.97 \pm 0.01$ & $0.59 \pm 0.01$ & $0.97 \pm 0.01$ & $5.65 \pm 0.02$ & $0.92 \pm 0.01$ & $0.72 \pm 0.01$ \\
\hline \multicolumn{7}{|l|}{ Men } \\
\hline $\mathrm{GX}$ & $1.93 \pm 0.01^{c}$ & $0.56 \pm 0.01^{\mathrm{a}}$ & $1.03 \pm 0.01$ & $5.72 \pm 0.03^{a}$ & $0.98 \pm 0.01^{\mathrm{b}}$ & $0.75 \pm 0.01^{b}$ \\
\hline $\mathrm{AA}$ & $1.99 \pm 0.01$ & $0.54 \pm 0.01$ & $0.99 \pm 0.02$ & $5.62 \pm 0.04$ & $0.94 \pm 0.01$ & $0.72 \pm 0.01$ \\
\hline \multicolumn{7}{|l|}{ Women } \\
\hline $\mathrm{GX}$ & $1.94 \pm 0.01$ & $0.62 \pm 0.00$ & $0.97 \pm 0.01$ & $5.67 \pm 0.01$ & $0.91 \pm 0.01$ & $0.71 \pm 0.00$ \\
\hline $\mathrm{AA}$ & $1.96 \pm 0.01$ & $0.63 \pm 0.01$ & $0.97 \pm 0.01$ & $5.68 \pm 0.02$ & $0.91 \pm 0.01$ & $0.71 \pm 0.01$ \\
\hline \multicolumn{7}{|l|}{ Model 2} \\
\hline \multicolumn{7}{|l|}{ All } \\
\hline $\mathrm{GX}$ & $1.94 \pm 0.01^{c}$ & $0.60 \pm 0.00$ & $0.99 \pm 0.01^{\mathrm{a}}$ & $5.69 \pm 0.01$ & $0.93 \pm 0.00$ & $0.72 \pm 0.00$ \\
\hline $\mathrm{AA}$ & $1.97 \pm 0.01$ & $0.59 \pm 0.01$ & $0.97 \pm 0.01$ & $5.65 \pm 0.02$ & $0.92 \pm 0.01$ & $0.72 \pm 0.01$ \\
\hline \multicolumn{7}{|l|}{ Men } \\
\hline $\mathrm{GX}$ & $1.93 \pm 0.01^{c}$ & $0.56 \pm 0.01^{\mathrm{a}}$ & $1.03 \pm 0.01^{\mathrm{a}}$ & $5.72 \pm 0.03^{a}$ & $0.98 \pm 0.01^{b}$ & $0.75 \pm 0.01^{b}$ \\
\hline $\mathrm{AA}$ & $1.99 \pm 0.01$ & $0.54 \pm 0.01$ & $0.98 \pm 0.02$ & $5.60 \pm 0.04$ & $0.94 \pm 0.01$ & $0.72 \pm 0.01$ \\
\hline \multicolumn{7}{|l|}{ Women } \\
\hline $\mathrm{GX}$ & $1.94 \pm 0.01$ & $0.62 \pm 0.00$ & $0.97 \pm 0.01$ & $5.67 \pm 0.01$ & $0.91 \pm 0.01$ & $0.71 \pm 0.00$ \\
\hline $\mathrm{AA}$ & $1.96 \pm 0.01$ & $0.63 \pm 0.01$ & $0.96 \pm 0.01$ & $5.67 \pm 0.02$ & $0.91 \pm 0.01$ & $0.71 \pm 0.01$ \\
\hline \multicolumn{7}{|l|}{ Model 3} \\
\hline \multicolumn{7}{|l|}{ All } \\
\hline $\mathrm{GX}$ & - & - & - & - & $0.93 \pm 0.00$ & $0.72 \pm 0.00$ \\
\hline $\mathrm{AA}$ & - & - & - & - & $0.92 \pm 0.01$ & $0.72 \pm 0.01$ \\
\hline \multicolumn{7}{|l|}{ Men } \\
\hline $\mathrm{GX}$ & - & - & - & - & $0.97 \pm 0.01^{\mathrm{b}}$ & $0.75 \pm 0.01^{b}$ \\
\hline $\mathrm{AA}$ & - & - & - & - & $0.94 \pm 0.01$ & $0.72 \pm 0.01$ \\
\hline \multicolumn{7}{|l|}{ Women } \\
\hline $\mathrm{GX}$ & - & - & - & - & $0.91 \pm 0.01$ & $0.71 \pm 0.00$ \\
\hline $\mathrm{AA}$ & - & - & - & - & $0.91 \pm 0.01$ & $0.72 \pm 0.01$ \\
\hline \multicolumn{7}{|l|}{ Model 4} \\
\hline \multicolumn{7}{|l|}{ All } \\
\hline $\mathrm{GX}$ & - & - & - & - & $0.93 \pm 0.00$ & $0.72 \pm 0.00$ \\
\hline $\mathrm{AA}$ & - & - & - & - & $0.92 \pm 0.01$ & $0.72 \pm 0.01$ \\
\hline \multicolumn{7}{|l|}{ Men } \\
\hline $\mathrm{GX}$ & - & - & - & - & $0.98 \pm 0.01^{b}$ & $0.75 \pm 0.01^{b}$ \\
\hline $\mathrm{AA}$ & - & - & - & - & $0.94 \pm 0.01$ & $0.73 \pm 0.01$ \\
\hline \multicolumn{7}{|l|}{ Women } \\
\hline $\mathrm{GX}$ & - & - & - & - & $0.91 \pm 0.01$ & $0.71 \pm 0.00$ \\
\hline $\mathrm{AA}$ & - & - & - & - & $0.91 \pm 0.01$ & $0.71 \pm 0.01$ \\
\hline
\end{tabular}

${ }^{\mathrm{a}} \mathrm{p}<0.05,{ }^{\mathrm{b}} \mathrm{p}<0.01,{ }^{\mathrm{c}} \mathrm{p}<0.001 \mathrm{vs}$. AA, $\mathrm{p}$-values were derived from ANCOVA test. Model 1: adjusted for age and BMI, Model 2: log TG and HDL-C were adjusted for age, $\mathrm{BMI}, \mathrm{SBP}$, and $\mathrm{HbA}_{1 \mathrm{c}}$; Glu and $\mathrm{HbA}_{1 \mathrm{c}}$ were adjusted for age, $\mathrm{BMI}, \mathrm{SBP}$ and log TG; max CIMT and mean CIMT were adjusted for age, BMI, SBP, $\log \mathrm{TG}$, and $\mathrm{HbA}_{1 \mathrm{c}}$, Model 3: adjusted for age, BMI, SBP, $\log \mathrm{TG}, \mathrm{HbA}_{1 \mathrm{c}}$, and smoking status, Model 4: adjusted for age, $\mathrm{BMI}, \mathrm{SBP}, \log \mathrm{TG}, \mathrm{HbA}_{1 \mathrm{c}}$, smoking status and history of current medications for $\mathrm{HT}, \mathrm{DM}$, and DL.

Interestingly, sex-dependent findings were demonstrated in this study, since the association between the GCKR rs780094 and metabolic traits including CIMT was found only in men. In general, sex is known to affect risks of nearly all of the common diseases, including atherosclerosis, DM, and their preceding risk factors. It has been reported that several SNPs of genes that have protective effects against atherosclerotic disease, such as $A P O E$ and
$A P O A 1$, have gene-environmental interactions by sex [23]. Since these genes, as well as GCKR, are associated with lipid metabolism, the different effects by sex may be due to the difference between men and women in lipid metabolism.

Age-related progression of CIMT has indeed been shown to be different between men and women. The protective effects of estrogen against atherosclerosis are well 
known [24], but Kozàkovà et al. reported that the differences in CIMT between men and women were significant in the 4th and 5th decades, but not in the 6th decade, when stratified by age decade [25]. Since $76 \%$ of the participants in the present study were older than 60 years, the different effect of GCKR rs780094 on progression of CIMT could not be fully explained by the protective effect of estrogen alone. The present results are consistent with the general concept of sex-dependency. Since the effect of SNP alone in a multifactorial disease is small, we should consider the effects of other genetic factors, as well as innumerable environmental and behavioral factors, on the relationship between GCKR rs780094 and CIMT. Further studies are needed.

There were significant differences in CIMT only between AA and GA. However, relative but not significant differences in maximum and mean CIMTs were observed between AA and GG, which suggests that the relatively small allele numbers of GG might cause the different effects.

The precise molecular mechanism by which the GCKR rs780094 relates to the clinical phenotype, including glucose and lipid metabolism and progression of CIMT, has seldom been investigated. However, the molecular mechanism has been partially clarified by which GCKR rs1260326 (P446L) variant, an SNP with a strong linkage disequilibrium to GCKR rs780094, is related to glucose and lipid metabolism [26]. Wild type GCKR is supposed to bind GCK and inhibit GCK enzyme activity in the nucleus of the liver at a physiological concentration of fructose6-phosphate (F6P), while the GKRP P446L is likely to dissociate with GCK even at the same concentration of F6P $[27,28]$. This means that the GKRP P446L may provide for increased activity of GCK compared to wild-type GKRP. GCK enhances glycolysis and promotes de novo lipogenesis in the liver [26]. With the variant of GKRP P446L, blood glucose and insulin levels would be reduced as a result of the enhancement of hepatic glucose disposal by increased GCK. The increase in liver GCK activity leads to increased glycolysis and activation of carbohydrate response element binding protein, which causes increased liver lipogenesis and secretion of TG [29]. This seems to be the mechanism by which GCKR rs1260326 is associated with increased synthesis of TG. Since the clinical phenotypes associated with GCKR rs1260326 and those with GCKR rs780094 are very similar, GCKR rs780094 might have a similar effect on GKRP function in the GKRP-GCK interaction.
Several limitations of this study warrant mention. In this study, Japanese adults ranging in age from 29 to 94 years who participated in the medical screening program were enrolled. The study participants were mainly middle-aged to elderly, community-dwelling subjects. Overall, $93 \%$ of the participants were more than 50 years old, and $76 \%$ of the participants were more than 60 years old. Therefore, this cohort cannot be representative of the entire community-dwelling population, since employees underwent their medical checkups at their workplaces in Japan. A relatively large sample size might detect the small associations between GCKR rs780094 and CIMT and laboratory findings. Furthermore, information regarding lifestyles, such as alcohol consumption, dietary habits, and physical activities, could not be collected. Thus, possible gene-environmental interactions could not be investigated. In addition, GCKR rs1260326 was not genotyped. Since GCKR rs1260326 is known to have strong linkage disequilibrium with GCKR rs780094, the present findings might not be solely associated with GCKR rs780094. The present findings may have been at least partially affected by GCKR rs1260326, synergistically or additively.

In conclusion, GCKR rs780094 was associated with TG, HDL-C, and $\mathrm{HbA}_{1 \mathrm{c}}$ levels, as well as with CIMT in Japanese community-dwelling men, but not women, which suggest that the phenotype effect of GCKR rs780094 might be sex-dependent. Further studies are needed to clarify the influence of sex on the effects of the GCKR rs780094 genotype.

Acknowledgments: This study was supported by a Grantin-Aid from the Japan Society for the Promotion of Science (No. 21590699).

\section{Conflict of interest statement}

Authors' conflict of interest disclosure: The authors stated that there are no conflicts of interest regarding the publication of this article.

Research funding: None declared. Employment or leadership: None declared. Honorarium: None declared.

Received February 4, 2013; accepted August 4, 2013; previously published online August 29, 2013 


\section{References}

1. Van Schaftingen E, Detheux M, Veiga da Cunha M. Short-term control of glucokinase activity: role of a regulatory protein. FASEB J 1994;8:414-9.

2. Massa ML, Gagliardino JJ, Francini F. Liver glucokinase: an overview on the regulatory mechanisms of its activity. IUBMB Life 2011;63:1-6.

3. Iynedjian PB. Molecular physiology of mammalian glucokinase. Cell Mol Life Sci 2009;66:27-42.

4. Sparsø T, Andersen G, Nielsen T, Burgdorf KS, Gjesing AP, Nielsen AL, et al. The GCKR rs780094 polymorphism is associated with elevated fasting serum triacylglycerol, reduced fasting and OGTT-related insulinaemia, and reduced risk of type 2 diabetes. Diabetologia 2008;51:70-5.

5. de la Iglesia N, Mukhtar M, Seoane J, Guinovart JJ, Agius L. The role of the regulatory protein of glucokinase in the glucose sensory mechanism of the hepatocyte. J Biol Chem 2000;275:10597-603.

6. Chu CA, Fujimoto Y, Igawa K, Grimsby J, Grippo JF, Magnuson MA, et al. Rapid translocation of hepatic glucokinase in response to intraduodenal glucose infusion and changes in plasma glucose and insulin in conscious rats. Am J Physiol Gastrointest Liver Physiol 2004;286:G627-34.

7. Vaxillaire M, Cavalcanti-Proença C, Dechaume A, Tichet J, Marre M, Balkau B, et al. The common P446L polymorphism in GCKR inversely modulates fasting glucose and triglyceride levels and reduces type 2 diabetes risk in the DESIR prospective general French population. Diabetes 2008; 57:2253-7.

8. Ridker PM, Pare G, Parker A, Zee RY, Danik JS, Buring JE, et al. Loci related to metabolic-syndrome pathways including LEPR, HNF1A, IL6R and GCKR associate with plasma C-reactive protein: the Women's Genome Health Study. Am J Hum Genet 2008;82:1185-92.

9. Speliotes EK, Yerges-Armstrong LM, Wu J, Hernaez R, Kim LJ, Palmer CD, et al. Genome-wide association analysis identifies variants associated with nonalcoholic fatty liver disease that have distinct effects on metabolic traits. PLoS Genet 2011;7:e1001324.

10. Dupuis J, Langenberg C, Prokopenko I, Saxena R, Soranzo N, Jackson AU, et al. New genetic loci implicated in fasting glucose homeostasis and their impact on type 2 diabetes risk. Nat Genet 2010;42:105-16.

11. Qi Q, Wu Y, Li H, Loos RJ, Hu FB, Sun L, et al. Association of GCKR rs780094, alone or in combination with GCK rs1799884, with type 2 diabetes and related traits in a Han Chinese population. Diabetologia 2009;52:834-43.

12. Bi M, Kao WH, Boerwinkle E, Hoogeveen RC, RasmussenTorvik LJ, Astor BC, et al. Association of rs780094 in GCKR with metabolic traits and incident diabetes and cardiovascular disease: the ARIC Study. PLoS One 2010;5:e11690.

13. Onuma H, Tabara Y, Kawamoto R, Shimizu I, Kawamura $\mathrm{R}$, Takata Y, et al. The GCKR rs780094 polymorphism is associated with susceptibility of type 2 diabetes, reduced fasting plasma glucose levels, increased triglycerides levels and lower HOMA-IR in Japanese population. J Hum Genet 2010;55:600-4.

14. Yang Z, Wen J, Tao X, Lu B, Du Y, Wang M, et al. Genetic variation in the GCKR gene is associated with non-alcoholic fatty liver disease in Chinese people. Mol Biol Rep 2011;38:1145-50.

15. Johansen CT, Hegele RA. Genetic bases of hypertriglyceridemic . phenotypes. Curr Opin Lipidol 2011;22:247-53.

16. Mohás M, Kisfali P, Járomi L, Maász A, Fehér E, Csöngei V, et al. GCKR gene functional variants in type 2 diabetes and metabolic syndrome: do the rare variants associate with increased carotid intima-media thickness? Cardiovasc Diabetol 2010;9:79.

17. O'Leary DH, Polak JF, Kronmal RA, Manolio TA, Burke GL, Wolfson SK. Carotid-artery intima and media thickness as a risk factor for myocardial infarction and stroke in older adults. Cardiovascular Health Study Collaborative Research Group. N Engl J Med 1999;340:14-22.

18. Vigili de Kreutzenberg S, Tiengo A, Avogaro A. Cerebrovascular disease in diabetes mellitus: the role of carotid intima-media thickness. Nutr Metab Cardiovasc Dis 2009;19:667-73.

19. Lorenz MW, Markus HS, Bots ML, Rosvall M, Sitzer M. Prediction of clinical cardiovascular events with carotid intima-media thickness: a systematic review and meta-analysis. Circulation 2007;115:459-67.

20. Friedewald WT, Levy RI, Fredrickson DS. Estimation of the concentration of low-density lipoprotein cholesterol in plasma, without use of the preparative ultracentrifuge. Clin Chem 1972;18:499-502.

21. Kashiwagi A, Kasuga M, Araki E, Oka Y, Hanafusa T, Ito H, et al. International clinical harmonization of glycated hemoglobin in Japan: from Japan Diabetes Society to National Glycohemoglobin Standardization Program values. J Diabetes Invest 2012;3:39-40.

22. Stančáková A, Paananen J, Soininen P, Kangas AJ, Bonnycastle LL, Morken MA, et al. Effects of 34 risk loci for type 2 diabetes or hyperglycemia on lipoprotein subclasses and their composition in 6,580 nondiabetic Finnish men. Diabetes 2011;60:1608-16.

23. Ordovas JM. Gender, a significant factor in the cross talk between genes, environment, and health. Gend Med 2007;4(Suppl B):S111-22.

24. Mendelsohn ME, Karas RH. The protective effects of estrogen on the cardiovascular system. New Engl J Med 1999;340:1801-11.

25. Kozàkovà M, Palombo C, Morizzo C, Nolan JJ, Konrad T, Dekker JM, et al. Gender-specific differences in carotid intima-media thickness and its progression over three years: a multicenter European study. Nutr Metab Cardiovasc Dis 2013;23:151-8.

26. Beer NL, Tribble ND, McCulloch LJ, Roos C, Johnson PR, Orho-Melander M, et al. The P446L variant in GCKR associated with fasting plasma glucose and triglyceride levels exerts its effect through increased glucokinase activity in liver. Hum Mol Genet 2009;18:4081-8.

27. Rees MG, Ng D, Ruppert S, Turner C, Beer NL, Swift AJ, et al. Correlation of rare coding variants in the gene encoding human glucokinase regulatory protein with phenotypic, cellular, and kinetic outcomes. J Clin Invest 2012;122:205-17.

28. Rees MG, Wincovitch S, Schultz J, Waterstradt R, Beer NL, Baltrusch S, et al. Cellular characterisation of the GCKR P446L variant associated with type 2 diabetes risk. Diabetologia 2012;55:114-22.

29. Agius L. High-carbohydrate diets induce hepatic insulin resistance to protect the liver from substrate overload. Biochem Pharmacol 2013;85:306-12. 
Copyright of Clinical Chemistry \& Laboratory Medicine is the property of De Gruyter and its content may not be copied or emailed to multiple sites or posted to a listserv without the copyright holder's express written permission. However, users may print, download, or email articles for individual use. 